\title{
Modeling and Optimization of a Multi-Objective Ridesharing Problem in the Case of Medical Waste
}

\author{
Mustapha Ahlaqqach, Jamal Benhra, Salma Mouatassim,SafiaLamrani
}

\begin{abstract}
Medical wastes is now a major concern of the world community and more particularly that of Moroccans. Indeed, these wastes, classified as hazardous products, are the source of serious infections, contamination of groundwater and air pollution. Through this paper, we encouraged the use of ridesharing to cope with the risks and costs arising from the logistics of these medical wastes. Thus, we have proposed a mathematical model that governs the multi-objective nature of this logistics and the various constraints associated with it. Since the exact approach had difficulties in large instances, we proposed the Genetic Algorithm and Evolution Strategy as metaheuristic to solve the model. The Evolution Strategy showed its efficiency and stability and therefore we have demonstrated through this metaheuristic the possibility of a compromise between the main objectives of our model.
\end{abstract}

Keywords: Multi-objective optimization, Ridesharing, Pickup and delivery, Heterogeneous fleet, Reverse Logistic, HeuristicLab

\section{INTRODUCTION}

The medical waste (MW) are defined as those generated by medical care activities. According to the World Health Organization (WHO), they include infectious waste, anatomical waste, pointed and sharp objects, chemicals, genotoxic waste, and radioactive waste [1]. Hospitals, health care facilities, laboratories and research centers are the main sources of these wastes. The seriousness of the potential risks stemming from poor management of these wastes has made management of solid wastes in general, and MW in particular, one of the pillars of the United Nations Development Program [2]. Morocco is no exception to this international trend. As an important component in the climate system, the management of MW has caused a great deal of ink from civil society and the local press. Consequently, the Moroccan state has made a legal and technical effort to improve the management of MW. However, the current situation reveals a dramatic gap between regulation and practice in the field [3]. A considerable amount of recommendations has been cited in the GIZ report. Among

these recommendations, we find the outsourcing of collection

Revised Version Manuscript Received on August 19, 2019.

Mustapha AHLAQQACH, PhD Student: LRI, OSIL Team, ENSEM, CELOG-ESITH, Casablanca, Morocco, North America.(email: : ahlaqqach@gmail.com)

Jamal BENHRA, Research Director:LRI, OSIL Team, ENSEM, Casablanca, Morocco, North America.(email: : jbenhra@hotmail.com)

Salma MOUATASSIM, PhD Student:LRI , OSIL Team, ENSEM Casablanca, Morocco, North America.(email: :mouatassimsalma@gmail.com)

Safia LAMRANI, PhD Student:LRI , OSIL Team, ENSEM Casablanca, Morocco, , North America.(email: : lamranisafia@yahoo.com) and transport to professionals. Nevertheless, the absence of logistic operators makes this recommendation almost impossible. This situation has pressed the medical instances to keep their own fleets to transport their MW as shown in figure 1. However, this in-sourcing transport begins to be more and more expensive, mainly in urban areas such as the city of Casablanca where traffic is very intense. Thus, the clinics are looking for an alternative solution to control their cost while waiting for the conditions of outsourcing to be met in the Moroccan case.

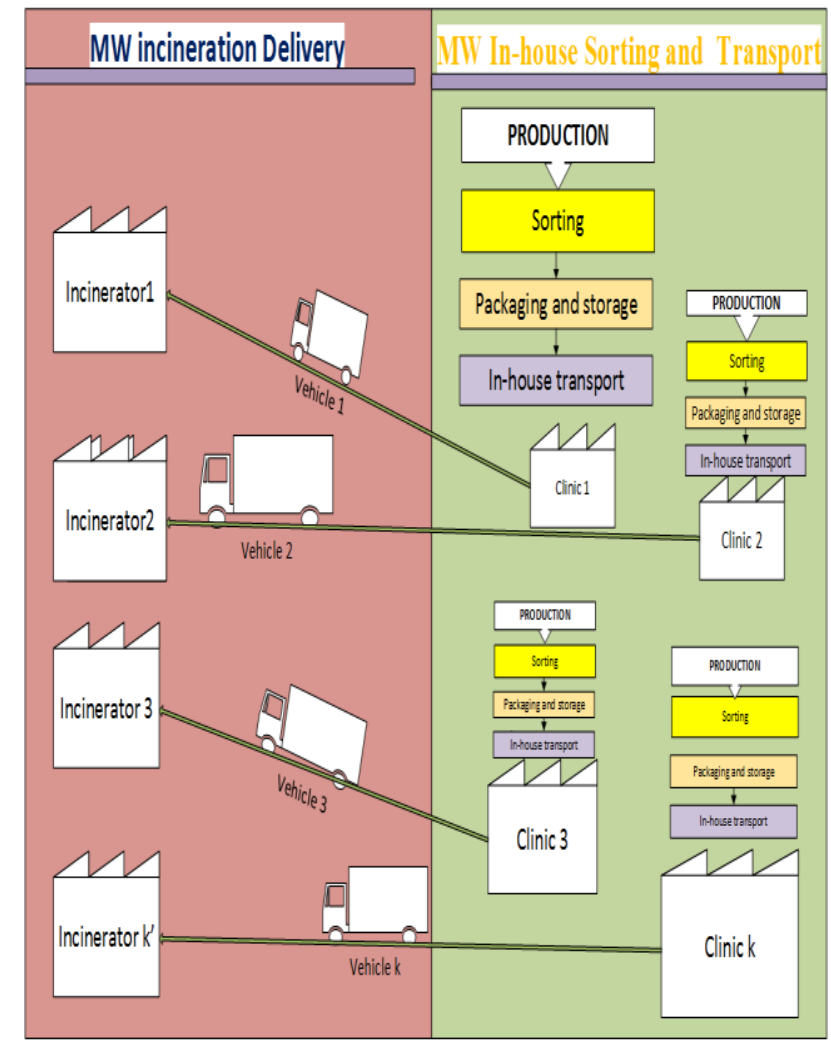

Figure 1. The Current Scheme for the Waste Disposal Management

Recently, several articles in the literature have clarified the benefits of ridesharing as an effective solution to control costs and pollution. The objective of this work is to study how to implement this solution for transportation of MW in the case of Casablanca city. To do this, the second section of 
this paper begins with a detailed definition of the problem and outlines the state of the art of the concepts linked to our problem. The remaining part of the paper proceeds as follows: The third part presents the mathematical model that governs the proposed model.

The fourth part develops the model resolution and carries out a series of experiments to test the robustness of the model and show its economic and environmental interest.

\section{PROBLEM DEFINITION AND STATE OF ART\& RESULTS}

As specified above, our aim objective is to benefit from ridesharing features in order to ease transport cost of clinics. Ridesharing is defined as a mode of transportation in which several travelers share a vehicle for a trip and split the trip costs[4]. several research studies have shown that ridesharing will not only generate economic gain but also protect environment and mitigate a traffic congestion[5][6]. These results encourage us to implement ridesharing in our case. In fact, the nature of the product transported is hazardous and can have a negative impact on the environment[7]. As we assume that incinerators (MW Delivery) and clinics (MW Collect) are going to be served by heterogeneous vehicles while respecting TW, our problem is called Heterogeneous capacitated Vehicle Routing Problem Pick-up and Delivery with time windows (HCVRPPDTW). Cherkesly et al. [8] proposed two different branch-price-and-cut algorithms to solve models and algorithms for the PDVRPTW and multiple stacks. This algorithm, applied in case of the transportation of heavy or dangerous material, gives good results with instances up to 75 according to the authors. Certainly, starting the project by integrating the elements cited above will lead to coalitions. Therefore, the potential risk of contamination from the coalition of clinics generating different categories of MW must be considered.

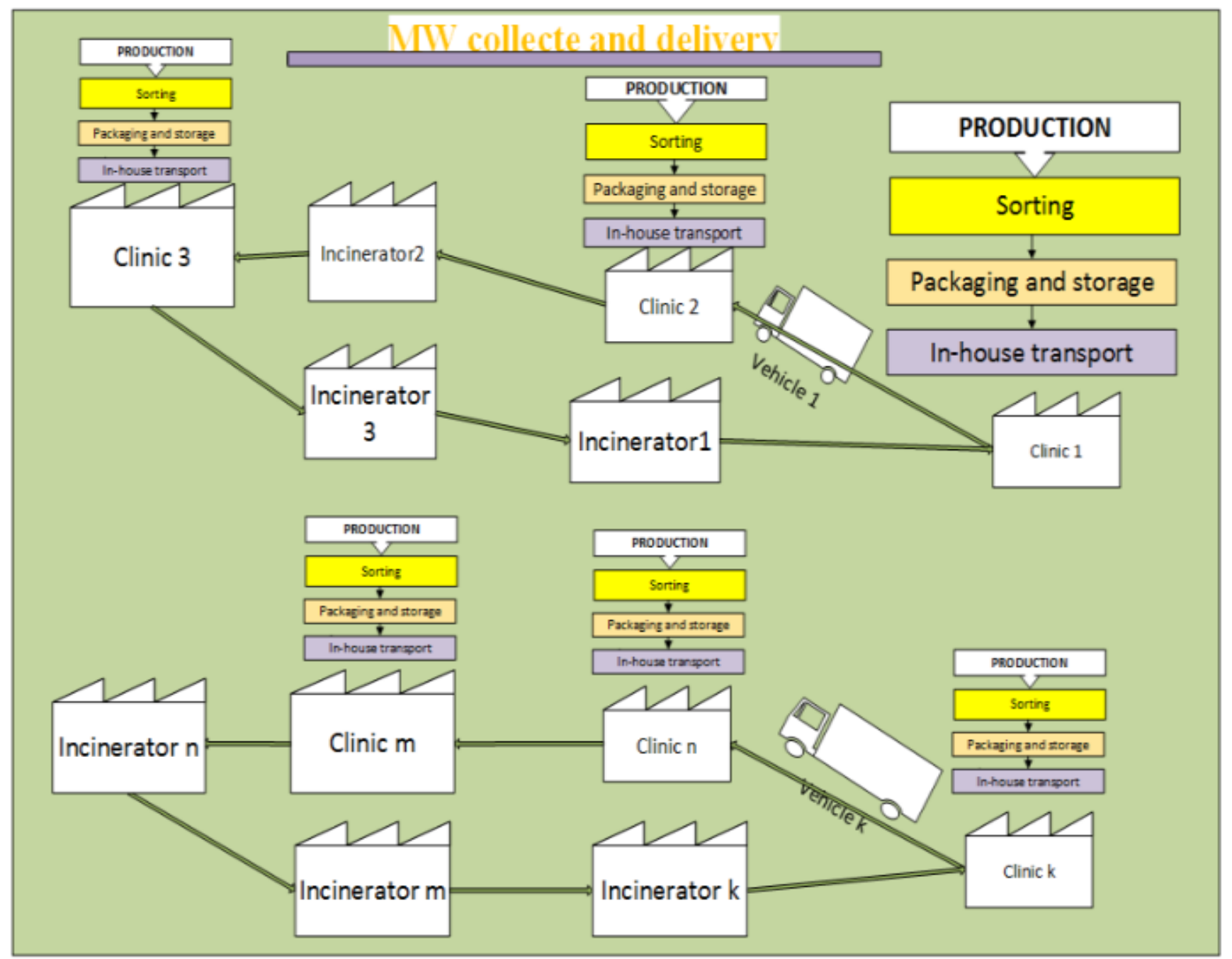

Figure 2. Proposed Ridesharing HCVRPPDTW Scheme for the MW Logistic's Management

Ridesharing (RS). Then, the authors can name the model

Roughly speaking our case is a ridesharing multi-objective case (cost, coalition risk) with HCVRPPDTW as shown in figure 02 . The present study fills a gap in the literature by adding some particularities compared to the existing models: Multi-objective (MO) model, Heterogeneous fleet (HF), Multiple Depot (MD), Strict Time Windows (TW), Hazardous Materials (MW), Pickup and delivery (PD) and studied a Ridesharing Multi-objective HazMat HCVRPPDTW (RMOHHFCVRPPDTW). In our knowledge, it is the first time that such model was studied. 
The reader should bear in mind that the study is based on a model where sorting takes place in-house. The distances between clinics and incinerators are symmetrical.

\section{MODEL FORMULATION}

\section{1) Hfcvrppdtw model:}

The HFCVRPPDTW can be defined on a directed graph $\mathrm{G}$ $=(\mathrm{N}, \mathrm{A})$, where: $\mathrm{N}=\{0, \ldots \mathrm{n}-1, \mathrm{n}, \ldots, 2 \mathrm{n}-1,2 \mathrm{n}, \ldots, 3 \mathrm{n}-1\}$ is the set of nodes and $A$ is the set of arcs where $n=$ the number of clinics $=$ the number of incinerators $=$ the number of virtual depots. The subsets $P=\{n, \ldots, 2 n-1\}$ is the sets of pickup (clinics) and delivery (incinerators). With each request $\mathrm{i}$ is associated a pickup node $\mathrm{i} \in \mathrm{P}$, where a request to pick up is strictly positive and a demand to deliver is null. Beside a delivery node $\mathrm{i} \in \mathrm{P}$, where a demand to deliver is strictly positive and a request to pick up is null. The parameters of our model are:

Dem : a demand to deliver in each node $i \in P$,

$$
\begin{aligned}
& \sum_{i=0}^{3 n-1} x_{i p k} \\
& =\sum_{j=0}^{3 n-1} x_{p j k} \\
& \in P, k \in K \\
& x_{0 k k} \\
& =y_{k} \\
& \in K \\
& x_{k+n 0 k} \\
& =y_{k} \\
& \in K \\
& x_{i i k}=0 \\
& \left(-1+x_{i 0 k}\right) * M \leq T_{i k}+s_{i}+t_{i 0}-T a_{k} \\
& \leq\left(1-x_{i 0 k}\right) * M \\
& i \in P, k \in K \\
& T_{i k}+s_{i}+t_{i j}+t w_{j}-T_{j k} \leq\left(1-x_{i j k}\right) * M i, j \in P, k
\end{aligned}
$$
$\leq\left(1-x_{0 j k}\right) * M$ $j \in P, k \in K$ )

$\mathrm{e}_{\mathrm{i}} \quad:$ the latest time at which service at node $\mathrm{i}$ can start $\mathrm{i} \in \mathrm{N}$,

$\mathrm{K}:$ : set of heterogeneous vehicles,

$b_{i} * y_{k} \leq T_{i k}$

$\leq e_{i} * y_{k}$

$i \in P, k$

Cfix : fixed cost if vehicle $\mathrm{k}$ is used,

$\mathrm{e}_{\mathrm{k}}$

Cvar : transport variable cost

$\in K$

$b_{0} * y_{k} \leq T a_{k}$

$\leq e_{0} * y_{k}$

$\in K$

$\mathrm{s}_{\mathrm{i}} \quad$ : service time at node $\mathrm{i}, \mathrm{i} \in \mathrm{N}$

$\mathrm{d}_{\mathrm{ij}} \quad$ : distance between node $\mathrm{i}$ and node $\mathrm{j},(\mathrm{i}, \mathrm{j}) \in \mathrm{N}$

$\mathrm{t}_{\mathrm{ij}} \quad$ : travel time between node $\mathrm{i}$ and node $\mathrm{j}$,

Decision variables:

$b_{0} * y_{k} \leq T d_{k}$

$\leq e_{0} * y_{k}$

$\in K$

$\left(-1+x_{i j k}\right) * M \leq G_{i k}-G_{j k}+D_{e m}$

$\mathrm{y}_{\mathrm{k}}$ : a binary variable (BV) equal to 1 if vehicle $\mathrm{k}$ is used, 0

$$
\leq\left(1-x_{i j k}\right) * M \quad i, j \in N, k \in K
$$$$
G_{j k}-c a_{k} * y_{k} \leq\left(1-x_{i j k}\right) * M \quad i \in N, j
$$
$\in_{\text {its }}$ $x_{i j k}, y_{k} \in\{0,1\} ; Z_{1}, t w_{i}, T_{i k}, T a_{k}, T d_{k}, G_{i k}$

$$
\in \mathbb{R}^{+} \quad i \in\{0 . . n\}, k \in K
$$

1.1. The equations (1)-(2) minimize the total travel cost depot node $\mathrm{k} \in \mathrm{K}$

$\mathrm{Z}_{1}$ : total cost of routing (Variable cost + fixed cost of u(fixed and variable) of the pickup and delivery vehicles vehicles)

The following equations manage our model:

Minimize $Z_{1}$

$Z_{1}=\sum_{k=0}^{K} y_{k} *$ Cfixe $_{k}+\sum_{i=0}^{3 n-1} \sum_{j=0}^{3 n-1} \sum_{k=1}^{K} x_{i j k} * \operatorname{Cvar}_{k} * d_{i j}$ subject to constraints (3) to (20). Constraints (3)-(4) assure that all pickup and delivery nodes are visited exactly once by the same vehicle. Flow conservation is considered by Constraints (5). Constraints (6)-(7) guarantee that each vehicle leaves its clinic at most once and its route starts and ends at its assigned clinic. The loop is avoided by the constraint (8). Constraints (9)-(14) compute the time variables and ensure that the time windows are respected,

$\sum_{i=0}^{3 n-1} \sum_{k=1}^{K} x_{i j k}$

$=1$

$\sum_{j=0}^{3 n-1} \sum_{k=1}^{K} x_{i j k}$

$j \in P$ also they avoid sub-tours. Constraints (15) computes the available MW in each vehicle and constraint (16)-(18) ensures that the capacity of each vehicle is respected at pickup and delivery nodes. Constraint (19) give the initial 
value of the load of vehicle at depot. Finally, constraint (20) defines the variables

\section{2)Risk Calculation: MOHHFCVRPPDTW model}

As aforementioned above all clinics insisted to compose homogeneous coalitions, particularly in term of risk contamination between clinics. In order to insure credibility and more pragmatism to our research, we start initial interviews with those clinics. The category of MW, proximity, relationship between clinics, rate of commitment to sustainable development and the respect of internal collect's standards are the main criterion cited to assess the risk contamination between clinics. Then, we give the set of criterion to the clinics to weight each criterion and fill their rating to be in coalition with another clinic according to each criterion. Based on the tables filled by clinics, we have established a risk matrix, which emphasize the risk contamination when two clinics are on the same coalition. We denote:

Risk $_{\mathrm{ij}}$ : the risk contamination between clinic $\mathrm{i}$ and clinic $\mathrm{j}$

$\mathrm{Z}_{\mathrm{ijk}} \quad$ a $\mathrm{BV}$ equal to 1 if clinic $\mathrm{i}$ and clinic $\mathrm{j}$ are on the same coalition using vehicle $\mathrm{k}, 0$ otherwise, $(\mathrm{i}, \mathrm{j}) \in \mathrm{P}, \mathrm{k} \in \mathrm{K}$

$\alpha, \beta$ : Weighting of cost function and risk function respectively

Then the risk calculation could be expressed as follow:

$$
Z_{2}=\sum_{i=n}^{2 n-1} \sum_{j=n}^{2 n-1} \sum_{k=1}^{K} z_{i j k} * R i s k_{i j}
$$

Subjected to:

$$
\begin{aligned}
& \sum_{p=0}^{3 n-1} x_{p i k}+\sum_{p=0}^{3 n-1} x_{p j k} \geq 2 * z_{i j k} \quad i, j \\
& \in\{n, \ldots, 2 n-1\}, i \neq j, k \in K \\
& \sum_{p=0}^{3 n-1} x_{p i k}+\sum_{p=0}^{3 n-1} x_{p j k} \leq 1+z_{i j k} \quad i, j \\
& \in\{n, \ldots, 2 n-1\}, i \neq j, k \in K
\end{aligned}
$$

The equation (22) will enforce $z_{i j k}=0$ if clinics $\mathrm{i}$ and $\mathrm{j}$ are not on the same coalition because the term in the left becomes less than 2. Whereas the equation (23) will enforce $z_{i j k}=1$ if clinics $i$ and $j$ are on the same coalition because the term in the left will be equal 2. As our model is multi-objective, the equation (1) becomes:

$$
\text { Minimize } \alpha * Z_{1}+\beta * Z_{2}
$$

3.3) Ridesharing constraints: RMOHHFCVRPPDTW model

The aim of our model is to limit the number of vehicle used by clinics in order to optimize cost and to protect the environment by limiting the $\mathrm{CO}_{2}$ emission. Roughly speaking, instead of using all vehicles, some clinics will engaged their vehicle to transport their MW and those of other clinics. Thus, we will enhance our model with constraints linked to Ridesharing.
Let:

$\mathrm{r}$ : a CV which represent node potentials indicating $i$ the visit order of node $i$ in the tour

The Rideshare model is subjected to the following constraints:

$$
\begin{aligned}
& \begin{array}{l}
x_{0 i k}=x_{i+n 0 k} \in\{n, \ldots, 2 n-1\}, k \in K \\
x_{0 i+n k}=x_{i 0 k}=0 \\
\sum_{j=0}^{3 n-1} x_{i j k}=\sum_{p=0}^{3 n-1} x_{i+n j k} \\
\quad \in\{n, \ldots, 2 n-1\}, k \in K
\end{array} \\
& \left.\begin{array}{l}
u_{i}-u_{j} \leq\left(1-x_{i j k}\right) * n-1 \\
u_{i}
\end{array} \quad i n, \ldots, 3 n-1\right\}, i \neq j, k \in K \\
& \leq u_{i+n} \\
& \in\{n, \ldots, 2 n-1\} \\
& u_{i} \\
& \geq 0 \\
& \in\{n, \ldots, 3 n-1\}
\end{aligned}
$$

Equation (25) make sure that a tour begins from the clinic, which own the vehicle used, and ends at his destination. However, constraint (26) prevent starting from incinerators or ending at clinics. Constraint (27) ensure the use of the same vehicle by origin and destination. Constraints (28) and (29) eliminate sub tours such as in equations (9) to (14) and ensure that each clinic precedes its corresponding incinerator. Finally, constraint (30) defines the non-negativity of $u_{i}$.

\section{4) Data of computational study:}

Set of clinics: The study concerns a set of 30 clinics disseminated in the whole region of Casablanca. Thanks to Google map we get addresses and coordinates of each clinic and the distance and travel time matrices. Analysis of the historical data from each clinic revealed the stability of the quantities of medical waste generated daily by each clinic. Thus, the demand of each clinic is the average number of daily MW's boxes generated. The distance between vehicle parking and waste storage in each clinic help us to estimate the unloading time. We have adopted a geographical breakdown based on the intensity of traffic. Consequently, areas with high traffic density should be served early before 8 am.

Set of vehicles: The size of the fleet is 30 vehicles and it is composed of 6 categories of vehicles with different capacities. The fixed and variable costs vary from one category to another.

\section{SOLUTION METHODOLOGY}

\section{1) Exact Approach:}

The validation of the model presented above is done on IBM ILOG CPLEX Optimization Studio 12.2. Our experiments are performed on an Intel ${ }^{\circledR}$ CORE(TM) i5-4200 CPU with a $2.3 \mathrm{Ghz}$ processor and $4 \mathrm{~GB}$ of RAM installed memory. The resolution by the exact approach has shown its 
limits when the search space is too large. The results coming from the exact approach will be used in the next section to compare them with those from the heuristic approach in small instance. Based on a very broad field of studies, the genetic algorithm (GA) and Evolutionary Strategy(ES) is promising approximation algorithm that has addressed hard problems in recent decades [9][10]. For this reason, they will be adopted in the heuristic approach.

\section{2) Heuristic Approach:}

ES was developed in the 70's by [11] and [12] at the technical university of berlin. The ES is mainly used at the first time to solve problems with continuous variables, discrete variants of ES are proposed to deal with combinatorial optimization problems. Recently Merkuryeva and V. Bolshakov[13] have studied integrated solutions for delivery planning and scheduling in distribution centers. The optimization using the ES gives the best solutions of a vehicle-scheduling problem with time windows. The GA is one of the best known evolutionary optimization techniques [14]. This meta-heuristic, which is based on the genetic method of the human body and the "elite of the strongest" in Darwin's theory, was initially developed by Holland [15] at the University of Michigan. Thangiah et al. [16] were the first to apply a GA to VRPTW. This first document describes an approach that uses a GA to find good coalition of customers. The main search operator in Genetic algorithms (GA) is the crossover operator which equally as significant as mutation, selection and coding in GA [17]. The crossover combines blocks from parents to produce their children. On the other hand, the mutation makes small local changes to ensure diversity in the population for a greater exploration of possible solutions. Several papers focus on crossover and mutation parameters for VRPTW [18] and others have worked on the improvement and comparison of crossover operators [19], [20] and [21]. HeuristicLab (HL) is a common platform, with a Graphical User Interface (GUI), equipped with heuristic and evolution algorithms, designed to use easily [22]. It combines both meta-heuristics and practical application cases whose interaction is possible thanks to the concept of the generic operator[23]. HL gives the user the possibility to define a new problem and realize the extension of the algorithms available in the library to adapt it to the context studied [24]. An extension of CVRPPDTW is written in $\mathrm{C} \#$ code to adapt to the studied RMOHHFCVRPPDTW. Based on preliminary experiments we established the best feasible results for each algorithm using the following parameters: ES (Children: 500, Maximum Generation: 400, Mutation operator: Alba Manipulator Parents Per Child: 10 Plus selection: Yes, $(\mu+\lambda)$-ES, Population Size: 10, Crossover operator:Potvin Crossover), AG (Crossover operator: Alba Crossover, Crossover Probability: 5\%, Elite: 5, Maximum Generation: 3000, Mutation operator: Alba Manipulator, Mutation Probability: 50\%, Population Size: 50, Selector: Proportional Selector). The validation of the GA and $\mathrm{ES}$ is done on the platform HL 3.3.15.15587.

\subsection{1) Validation and analysis of the approach:}

The benchmarking is the best way to validate the meta-heuristic proposed in the case of
RMOHHFCVRPPDTW. However, this is the first time that a problem of RMOHHFCVRPPDTW is dealt with in our knowledge. Nonetheless, we can generate our ES and GA for small instances to compare the results with the global optimum.

\section{Small Instance:}

The table 1 presents the comparison between the global optimum and the average results of heuristic's approach algorithm. Analyzing these results, we can conclude that the difference between the global optimum and the results of the proposed ES and GA are respectively of $6 \%$ and $11 \%$. The results encouraged us to justify the acceptability of the ES and GA algorithm's performance. Nonetheless,we noticed that ES gives better results compared to GA. Also, the resolution time of the heuristics approach is significantly better than that of the exact approach. As a result, both ES and GA are experimentally found to be fast and highly successful.

Table 1. Average results of different algorithm tested

$\begin{array}{llll}\text { Algorithm } & \begin{array}{l}\text { Objective } \\ \text { Function }\end{array} & \begin{array}{l}\text { GAP to Global } \\ \text { optimum }\end{array} & \begin{array}{l}\text { Execution } \\ \text { Time }\end{array} \\ \begin{array}{l}\text { Exact Approach } \\ 684\end{array} & & 23: 49,0 \\ \begin{array}{l}\text { Evolution } \\ \text { Strategy (ES) }\end{array} & 725 & 6 \% & 02: 38,2 \\ \begin{array}{l}\text { Genetic } \\ \text { Algorithm (GA) }\end{array} & 803 & 11 \% & 01: 05,1\end{array}$

\section{Real Instance:}

We will compare the performance of ES and GA regarding the treatment of the actual instance studied. The comparison is based on fifty tests for each algorithm. The results are pertinent and the GA finds the best result shown in table 2 . The ridesharing constraints chose clinics 5, 13 and 7 as a starting point. Therefore, their vehicles will be used to cover the routing according to the sequences specified in the table 2. The weakness of this solution is in the resolution time, which is quite high relative to the desired performance in this kind of problem. The average results of experiments, represented by table 3, showed that the performances of ES are absolutely higher than GA. Indeed, the quality given by the ES algorithm is $40 \%$ better than one given by GA. In addition, the ES resolution time is a reasonable time and insignificant compared to that of the GA. 
Table 2. Best result coming from real instance experiments

\begin{tabular}{|c|c|c|c|c|c|}
\hline & $\begin{array}{l}\text { Pickup and Delivery } \\
\text { Sequence }\end{array}$ & Cost & Risk & Quality & $\begin{array}{l}\text { Execution } \\
\text { Time }\end{array}$ \\
\hline $\begin{array}{l}\text { Routing } \\
1\end{array}$ & $\begin{array}{l}\text { C5 C2 C1 I19 I20 I } 23 \\
\text { C5 }\end{array}$ & 928,25 & 26,00 & 477,12 & \\
\hline $\begin{array}{l}\text { Routing } \\
2\end{array}$ & $\begin{array}{l}\text { C13 C10 C11 I29 I28 } \\
\text { C4 C3 I21 I22 } \\
\text { C9 I27 C15 C16 I34 I33 } \\
\text { I31 C13 }\end{array}$ & $3^{131}$ & 306,5 & 812,89 & \\
\hline $\begin{array}{l}\text { Routing } \\
3\end{array}$ & $\begin{array}{l}\text { C7 C14 I32 C6 I24 C12 } \\
\text { C8 I26 C17 } \\
\text { I30 C18 I36 I35 I25 C7 }\end{array}$ & 1084,42 & 218 & 651,21 & \\
\hline $\begin{array}{l}\text { C: Clin } \\
\text { Total: }\end{array}$ & c, I: Incinerator & 3331,95 & 550,5 & 1941,22 & $13: 23,3$ \\
\hline
\end{tabular}

Table 3. Average results of ES and GA in real instance

\begin{tabular}{|l|l|l|}
\hline & Best Quality & Execution Time \\
\hline Evolution Strategy & 2720.2 & $03: 43.1$ \\
\hline Genetic Algorithm & 3809.6 & $13: 05.9$ \\
\hline GAP ES/GA & $40 \%$ & \\
\hline
\end{tabular}

The results coming from small and large instance experiments confirm the validation of the selected ES and GA. Besides, the ES gave the best performances in a reasonable time. The following section will be dedicated to analyzing the stability of the proposed ES algorithm

\section{Population Size Experiments:}

To carry out this experiment we deal with two ES settings: population size " $10 "$ and " $40 "$ ". The results from this experiment are presented in Figure 3. The analysis of the results of the various tests reveals a difference between the quality of the instances ES (10) and ES (40). These negligible differences in quality lead the authors to conclude on the stability of the proposed ES, the accuracy of the parameterization procedure and the effectiveness of the results of the present study.

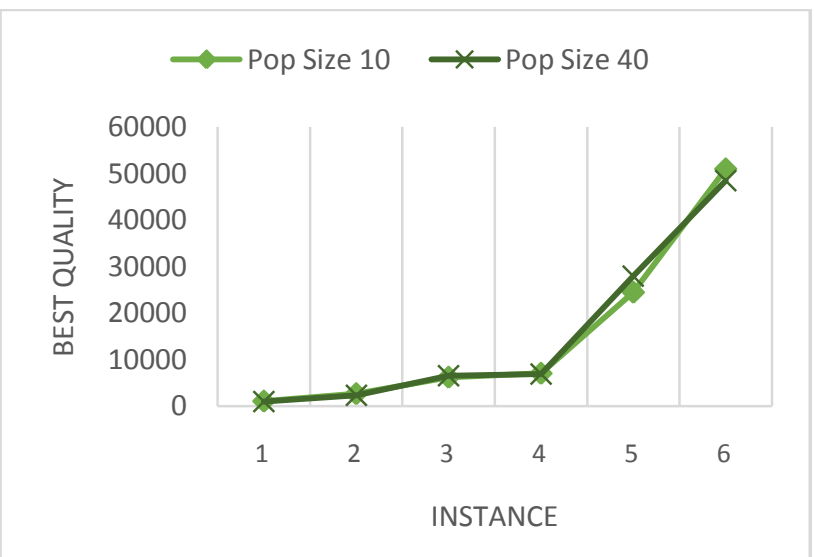

Figure 3.Best quality's Result of large-scale population size analysis

Experimentation of the weighting parameters:

As the ES has shown high efficiency in the experiments above, it was chosen to study the possibility of any compromise between cost and risk. To perform this experiment, we varied the weighting of $\alpha$ and $\beta$ to see the impact of the risk on the components of the objective function. Instead of using the absolutes values of $\mathrm{W}_{1}$ and $\mathrm{W}_{2}$, respectively, we normalize them so they become comparable. We use normalization in Bronfman et al. [25], where Yi is the normalized objective function. $\mathrm{W}_{\mathrm{imax}}, \mathrm{W}_{\mathrm{imin}}$ and $\mathrm{W}_{\mathrm{i}}$ represent the maximum, minimum and actual value of each objective before normalization.

$$
\mathbf{Y}_{\mathrm{i}}=\left[\frac{\mathbf{W}_{i}-\mathbf{W}_{\text {imin }}}{W_{\text {imax }}-W_{\text {imin }}}\right]
$$

As shown in Figure 4., the increase in the risk coefficient, from $\alpha<0.3$, leads to an exponential increase in routing costs. This behavior can be explained by the rejection of coalitions with a very high risk. So the model will choose more than one vehicle to build a low-risk coalition and therefore a more expensive route. An opposite behavior is observed for risk, the reinforcement of the cost coefficient, from $\alpha>=0.6$, leads to a better control of the routing cost and a spectacular increase in risk. The tuning of $\alpha$ and $\beta$ values makes it possible to locate the sensitivity area of the objective function. As shown in the figure 4., the components of the normalized objective function, cost and risk, are more stable and close to each other when $0.3<\alpha<0.6$. Thus, the search for any compromise between cost and risk must be done with $\alpha$ values in the interval ]0.3, 0.6[. In a practical case, clinics have to seek a compromise, which guarantees a desired coalition with an economic interest. Consequently, we can rely on our model to have such a compromise.

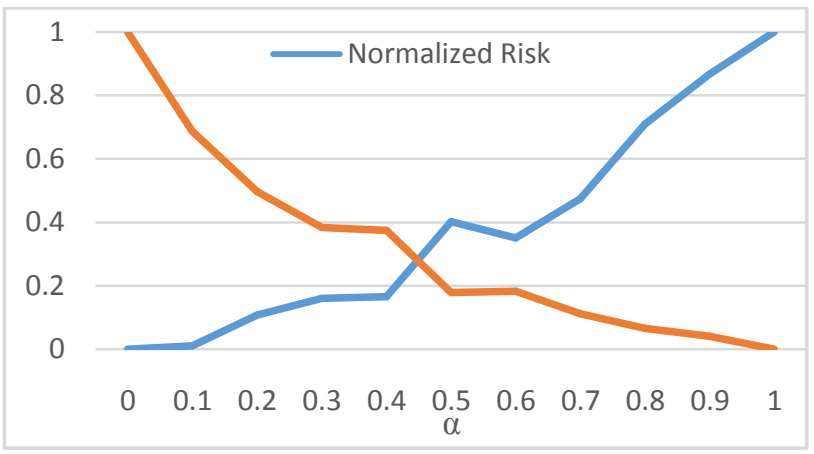

Figure 4.Evolution of the normalized objectives according to $\alpha$ value (Real case)

\section{CONCLUSION}

This research sheds new light on reverse logistics in the case of MW, by proposing a multi-objective ridesharing model with evolution algorithms of a very complicated case of HCVRPPDTW. Our aim was to find a solution to a problem facing humanity. To do this, we took into consideration several realistic characteristics of the problem in order to make the model close to reality. The potential risk coming from hazardous nature of the MW has led us to create homogeneous coalitions to encourage clinics to adhere the proposed ridesharing approach. As the exact approach showed its limits in large instance, we proposed two evolution algorithms: ES and GA to better understand this phenomenon of the problem size. The performances of these algorithms are highly acceptable in both small and large-scale. Nonetheless, for the large scale, the ES is more effective than GA; it gave the best solutions within a reasonable time and showed its stability. Consequently, we 
made a tuning of risk and cost parameters to study the possibility of any compromise in our multi-objective problem. The results showed that we can rely on our model to find a compromise between the different clinics.

The reader should bear in mind that the study assumed that the parameters are deterministic. Thus, for a better contribution in the framework of our scientific research, there is some guidance for future research. We will use non-deterministic approaches to deal with the nature of parameters. Also we will integrate the game theory approach to establish coalitions between clinics. In fact, This approach has been successful in creating such coalition (Mouatassim et al., 2016)

\section{REFERENCES}

1. OMS, "Organisationmondiale de la Santé | Les déchetsliés aux soins de santé," WHO, 2015. [Online]. Available: http://www.who.int/mediacentre/factsheets/fs253/fr/. [Accessed: 28-Feb-2016].

2. UNDP, "Chemicals and waste management | United Nations Development Programme," 2016. [Online]. Available:

http://www.undp.org/content/undp/en/home/ourwork/sus tainable-development/natural-capital-and-the-environme nt/chemicals-and-waste-management.html. [Accessed: 28-Feb-2016]

3. A. Mbarki, B. Kabbachi, A. Ezaidi, and M. Benssaou, "Medical Waste Management: A Case Study of the Souss-Massa-Drâa Region, Morocco," J. Environ. Prot. (Irvine,.Calif)., vol. 4, no. 2, pp. 914-919, 2013.

4. D. Sánchez, S. Martínez, and J. Domingo-Ferrer, "Co-utile P2P ridesharing via decentralization and reputation management," Transp. Res. Part C Emerg. Technol., vol. 73, pp. 147-166, Dec. 2016.

5. B. Caulfield, "Estimating the environmental benefits of ride-sharing: A case study of Dublin," Transp. Res. Part D Transp. Environ., vol. 14, no. 7, pp. 527-531, Oct. 2009.

6. N. D. Chan and S. A. Shaheen, "Ridesharing in North America: Past, Present, and Future," Transp. Rev., vol. 32, no. 1, pp. 93-112, Jan. 2012.

7. M. Ahlaqqach, J. Benhra, and S. Mouatassim, "Optimisation des tournées de collecte et de desserte des déchetsmédicauxtransitant par un entrepôtcommun," LogistiqueManag., vol. 25, no. 1, pp. 25-33, 2017.

8. M. Cherkesly, G. Desaulniers, S. Irnich, and G. Laporte, "Branch-price-and-cut algorithms for the pickup and delivery problem with time windows and multiple stacks," Eur. J. Oper. Res., vol. 250, no. 3, pp. 782-793, 2016.

9. R. Liu, X. Xie, V. Augusto, and C. Rodriguez, "Heuristic algorithms for a vehicle routing problem with simultaneous delivery and pickup and time windows in home health care," Eur. J. Oper. Res., vol. 230, no. 3, pp. 475-486, Nov. 2013.

10. H. Kanoh and S. Tsukahara, "Virus Evolution Strategy for

11. Vehicle Routing Problems with Time Windows," Springer, Berlin, Heidelberg, 2008, pp. 1041-1050.

12. I. Rechenberg, Evolutionsstrategie; OptimierungtechnischerSystemenachPrinzipien der biologischen Evolution.Frommann-Holzboog, 1973.

13. H.-P. Schwefel, NumerischeOptimierung von Computor-Modellenmittels der Evolutionsstrategie miteinervergleichendenEinführung in die Hill -Climbingund Zufallsstrategie. Birkhäuser, 1977.

14. G. Merkuryeva and V. Bolshakov, "Integrated Solutions for Delivery Planning and Scheduling in Distribution Centres," in Applied Simulation and Optimization, Cham: Springer International Publishing, 2015, pp. 135-168.
15. H. Soleimani, M. Seyyed-Esfahani, and M. A. Shirazi, "Designing and planning a multi-echelon multi-period multi-product closed-loop supply chain utilizing genetic algorithm," Int. J. Adv. Manuf. Technol., vol. 68, no. 1-4, pp. 917-931, 2013.

16. J. H. Holland, Adaptation in natural and artificial systems. Ann Arbor, MI, USA: University of Michigan Press, 1975.

17. S. R. Thangiah, K. E. Nygard, and P. L. Juell, "GIDEON a genetic algorithm system for vehicle routing with time windows," in The Seventh IEEE Conference on Artificial Intelligence Application, 1991, vol. i, pp. 322-328.

18. S. M. Lim, A. B. M. Sultan, M. N. Sulaiman, A Mustapha, and K. Y. Leong, "Crossover and Mutation Operators of Genetic Algorithms," Int. J. Mach. Learn. Comput., vol. 7, no. 1, pp. 9-12, Feb. 2017.

19. J.-Y. Potvin and S. Bengio, "1 The Vehicle Routing Problem with Time Windows - Part II: Genetic Search Jean-Yves Potvin," INFORMS J. Comput., vol. 8, no. 2, pp. 1-21, 1996.

20. A. Misevičius and B. Kilda, "Comparison of crossover operators for the quadratic assignment problem," Inf. Technol. Control, vol. 34, no. 2, pp. 109-119, 2005.

21. R. Kumar, N. Kumar, and Karambir, "A Comparative Analysis of PMX , CX and OX Crossover operators for solving Travelling Salesman Problem," Int. J. Latest Res. Sci. Technol., vol. 1, no. 2, pp. 98-101, 2012.

22. H. El Hassani, S. Benkachcha, and J. Benhra, "New Genetic Operator (Jump Crossover) for the Traveling Salesman Problem," Int. J. Appl. MetaheuristicComput. vol. 6, no. 2, pp. 33-44, 2015.

23. A. Scheibenpflug, A. Beham, M. Kommenda, J. Karder, S. Wagner, and M. Affenzeller, "Simplifying Problem Definitions in the HeuristicLab Optimization Environment," Proc. Companion Publ. 2015 Annu. Conf. Genet. Evol. Comput., pp. 1101-1108, 2015.

24. S. Wagner et al., "Architecture and Design of the HeuristicLab Optimization Environment," Springer, Heidelberg, 2014, pp. 197-261.

25. M. Ahlaqqach, J. Benhra, S. Mouatassim, and S. Lamrani, "Smart City Through the Control of the Healthcare Textiles Transport," SSRN Electron. J., May 2018.

26. A. Bronfman, V. Marianov, G. Paredes-Belmar, and A. Lüer-Villagra, "The maxisum and maximin-maxisum HAZMAT routing problems," Transp. Res. Part E Logist. Transp. Rev., vol. 93, pp. 316-333, Sep. 2016.

27. S. Mouatassim, M. Ahlaqqach, J. Benhra, and M ELOUALIDI, "Model based on hybridized game theory to optimize logistics case of blood supply chain," Int. J. Comput. Appl., vol. 145(15), no. 15, pp. 37-48, 2016. 


\section{AUTHORS}

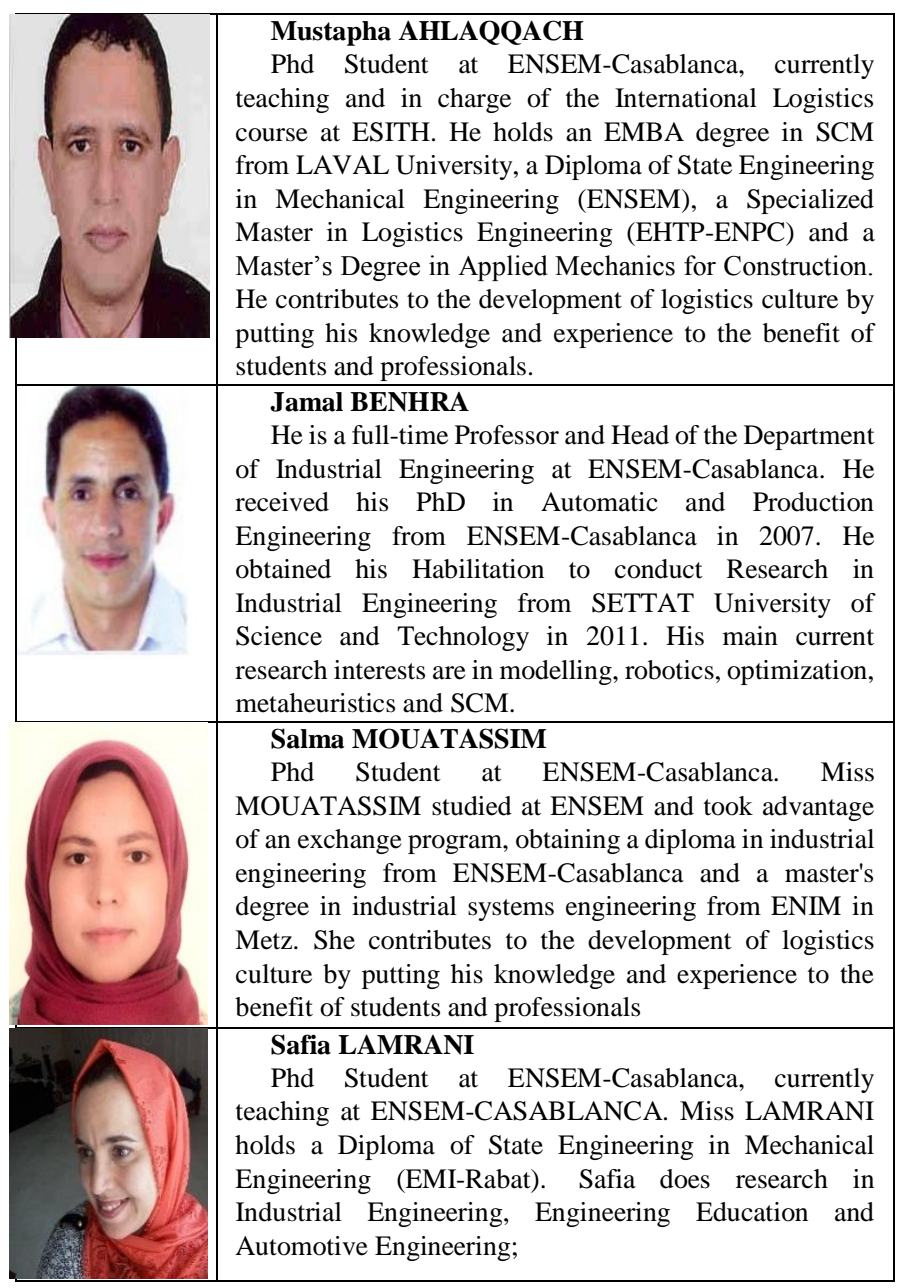

